\title{
Technology and the New Paradigm of Education
}

\author{
Charles M. Reigeluth \\ Indiana University, United States
}

The use of technology in education should be based on what we know about how students learn best and how we can best facilitate that learning. Tools should be selected based on purposes or functions to be served, not the other way around.

In our current educational system, student progress to a new topic is based on time, not on learning. If it is Monday, we move on to the next topic, in spite of some students not having attained the standards just taught. This system is sorting-focused (which was appropriate for the Industrial Age when manual labor was predominant), not learning-focused (which is needed for the Information Age when knowledge work is predominant. A learning-focused system would not allow a student to move on until she or he succeeded in attaining the current standard. And it would require each student to move on as soon as he or she succeeded in attaining the current standard. This requires a completely different paradigm of education, one that is customized to meet each student's needs and potential. This requires a different role for teachers, students, and, yes, technology.

Rather than integrating technology into the classroom (the current paradigm), we should be using technology to transform what goes on in the classroom. Technology allows us to customize education in a way that was not possible before. So how should technology be used? We currently see four main roles or functions for technology to make a customized, learning-focused educational system feasible and cost-effective. Each of these is described next.

Record keeping for student learning. Attainment-based progress for students requires a personal record of attainments for each student. This replaces the current report card, and it has three parts. First, it has a Standards Inventory that contains both required educational standards (national, state, and local) and optional educational standards for access by the teacher, student, and parents. It presents a list of things that should or can be learned, along with levels or standards or criteria at which they can be learned. Second, it has a Personal Attainments Inventory that contains a record of what each student knows. In essence, it maps each student's progress on the attainments listed in the Standards Inventory (and perhaps some that are not yet listed there). It shows when each attainment was reached, which ones are required, what the next required attainments are in each area, and links to evidence of each attainment (in the form of summary data and/or original artifacts). Third, it has a Personal Characteristics Inventory that keeps track of each student's characteristics that influence learning, such as learning styles, profile of multiple intelligences, student interests, and major life events. 
Planning for student learning. Customized education also requires a personal learning plan, or contract, for each student. It helps the student, parents, and teacher to (a) decide on long-term goals; (b) identify the full range of attainments that are presently within reach for the student; (c) select from those options the ones that they want to pursue now (short-term goals), based on requirements, long-term goals, interests, opportunities, etc.; (d) identify projects (or other means) for attaining the short-term goals; (e) identify other students who are interested in doing the same projects (if desired); (f) specify the roles that the teacher, parent, and any others might play in supporting the student in learning from the project; and (g) develop a contract that specifies goals, projects, teams, parent and teacher roles, and the deadline for each project.

Instruction for student learning. Trying to "instruct" 25 students who are all learning different things at any point in time could be very difficult for teachers if they had to be the agent of instruction all the time, as is typical in the Industrial-Age paradigm. However, we envision that learning will be primarily project (or problem) based, with both a project "space" and an instructional "space." In the problem space, technology offers an introduction to the project, resources for doing the project (including research tools and communication tools), and usually a place for working on the project. While working on the project, whenever the student encounters a deficiency in knowledge, skills, or understandings needed for doing the project, each student switches from the project space to his or her personalized instructional space, where highly efficient and appealing tools offer customized drill-and-practice, tutorials, simulations, and educational games, as appropriate, to foster the different kinds of learning in the most effective, efficient, and appealing way. The student continues to work in the instructional space until he or she reaches the criterion for mastery of the standard on authentic performances (based on appropriate amounts of transfer learning and automatization of skills), as described in the next section. In addition, technology provides tools for the teacher to monitor and support student progress on the project, and even provides tools to help teachers and others develop new projects and new instructional tools.

Assessment for (and of) student learning. Conducting formative and summative assessments of students could be a nightmare for teachers, since students are not all taking a given test at the same time. And once again, technology can offer great relief. First, assessment is integrated with instruction. The plentiful performance opportunities that are used to cultivate skills and understandings are used for both formative and summative assessments. Second, the assessments present authentic tasks on which the students demonstrate their knowledge, understanding, and skill. Third, whether in a simulation or a tutorial or drill and practice, the technology is designed to evaluate whether or not the criterion was met on each performance and to provide formative feedback immediately to the student for the greatest impact. When the criteria for successful performance have been met on $x$ out of the last $y$ performances, the summative assessment is complete and the corresponding attainment is automatically checked off in the student's personal inventory of attainments. In the few cases where the technology cannot assess the performance, an observer has a handheld device with a rubric for assessment and personally provides the immediate feedback on student performances. The information from the handheld device is uploaded into the computer system, where it is placed in the student's personal inventory. Finally, technology provides tools to help teachers develop assessments and link them to the standards. Instructional-evaluation theory is critical for technology to reach its potential contribution to this role. 
Note that these four roles or functions are seamlessly integrated. The record-keeping tool provides information automatically for the planning tool. The planning tool identifies instructional tools that are available. The assessment tool is integrated into the instructional tool. And the assessment tool feeds information automatically into the record-keeping tool. Also, please note that there are many other roles or functions for such a system. These secondary functions include communications (email, blogs, web sites, discussion boards, wikis, whiteboards, instant messaging, podcasts, videocasts, etc.), system administration (offering access to information and authority to input information based on role and information type), general student data (student's address, parent/guardian information, mentor-teacher and school, student's location/attendance, health information), school personnel information (address, certifications and awards, location, assigned students, tools authored, student evaluations that they have performed, teacher professional development plan and records, repository of teaching tools, awards their students have received), and more.

It should be apparent that technology will play a crucial role in the success of the Information-Age paradigm of education. It will enable a quantum improvement in student learning, and likely at a lower cost per student per year than in the current Industrial-Age paradigm. Just as the electronic spreadsheet made the accountant's job quicker, easier, and less expensive, the kind of system described here will make the teacher's job quicker, easier, and less expensive. However, the use of technology must be theory-driven to realize its potential contribution.

Correspondence: Charles M. Reigeluth, Professor, Instructional Systems Technology, School of Education, Indiana University, Bloomington, IN 47405, United States. 